\title{
La contraception d'urgence derrière le comptoir
}

$\mathrm{B}$ onne nouvelle, le lévonorgestrel, ou «pilule du lendemain», est reclassé par Santé Canada dans les médicaments non prescrits, ce qui permet aux pharmaciens de dispenser directement ce contraceptif oral postcoïtal aux femmes qui en ont besoin, au moment où elles en ont besoin $^{1}$ (voir page 861). Moins bonne nouvelle, les provinces classent le lévonorgestrel $0,75 \mathrm{mg}$ (Plan B) dans les médicaments vendus «derrière le comptoir», obligeant toute femme qui cherche ainsi à éviter une grossesse non désirée à consulter un pharmacien. Santé Canada appuie cette politique en affirmant que «les pharmaciens sont en mesure de jouer un rôle important pour (...) donner des conseils sur les différentes options de contraception ${ }^{2} \gg$.

Or, ce n'est pas nécessairement le cas. Même si les pharmaciens ont des contacts de première ligne avec les patientes et disposent de lignes directrices professionnelles sur la prestation de moyens de contraception d'urgence, ils et elles ne sont pas dans une situation idéale pour jouer un rôle de conseil dans leur contexte de pratique usuel. Peu de pharmacies offrent la discrétion nécessaire à la tenue d'une telle conversation. Ce simple fait, conjugué aux honoraires professionnels découlant de la consultation, constitue un obstacle inutile à l'accès.

De plus, la nécessité de consulter un professionnel pour obtenir un contraceptif oral d'urgence est douteuse. Le lévonorgestrel $0,75 \mathrm{mg}$ est disponible au Canada sur ordonnance depuis 2000. Une première dose prise dans les 72 heures suivant une relation sexuelle non protégée, suivie d'une deuxième dose 12 heures plus tard, réussit très efficacement à prévenir l'ovulation, la fécondation et l'implantation. Il n'y a pas d'effet indésirable sérieux pour la femme ou, s'il se trouve qu'elle soit déjà enceinte, le fœetus. De plus, le lévonorgestrel satisfait à tous les critères régissant le statut de médicament non prescrit, y compris une longue histoire d'utilisation sûre et efficace, une faible incidence d'effets secondaires (principalement de faibles nausées) et un mode d'emploi simple et facile à expliquer. Les études portant sur les contraceptifs oraux d'urgence autoadministrés ont révélé un taux élevé d'utilisation appropriée sans effet indésirable ${ }^{3,4}$. Pourquoi alors considérer les femmes capables qui ont eu un problème de contraception ou ont manqué de précaution, ou qui ont été victimes de cœercition ou d'une agression sexuelle, comme une cible facile de questions indésirées et de conseils non sollicités - à leurs propres frais?

En 2001, il s'est pratiqué plus de 106000 avortements au Canada, dont 20000 chez des femmes de moins de 20 ans. On estime que de 40 à $50 \%$ des grossesses au Canada ne sont pas désirées en dépit de la grande disponibilité des moyens de contraception. Le manque d'accès rapide à un médecin constitue l'un des obstacles importants à l'utilisation des contraceptifs oraux d'urgence. La disponibilité sans ordonnance dans les pharmacies de la «pilule du lendemain» constitue un grand pas en avant.

En Colombie-Britannique, en Saskatchewan et au Québec, la loi permet déjà aux pharmaciens de dispenser ce médicament sans ordonnance d'un médecin. En reclassant le médicament, Santé Canada contribue à l'équité d'accès aux contraceptifs d'urgence pour les femmes de toutes les régions du Canada. Dans ce numéro, Judith Soon et ses collaborateurs ${ }^{5}$ (voir page 878 ) présentent un rapport sur l'évolution de l'utilisation des contraceptifs d'urgence en C.-B. de 1996 à 2002. Les auteurs souhaitaient particulièrement déterminer s'il existe un lien entre la distribution sans ordonnance de contraceptifs d'urgence par les pharmaciens dans cette province (à compter de décembre 2000) et une diminution équivalente des ordonnances rédigées par les médecins. Cela n'a pas été le cas. Le nombre des ordonnances établies par les médecins a augmenté, de concert toutefois avec une montée spectaculaire de l'utilisation totale découlant principalement de la distribution par les pharmacies. Il y a donc eu augmentation nette de l'utilisation. Nous pouvons maintenant nous attendre à une diminution nette des taux d'avortement en C.-B. et dans tout le Canada. C'est une bonne nouvelle.

La nouvelle serait toutefois encore meilleure s'il ne persistait pas toujours, caché derrière le comptoir, un certain paternalisme dans les questions touchant la santé génésique des femmes. - $7 A M C$

\section{Références}

1. Sibbald B. Nonprescription status for emergency contraception. $7 A M C$ 2005;172(7):861-2.

2. Gouvernement du Canada. Règlement modifiant le Règlement sur les aliments et drogues (1272 - lévonorgestrel). C Gaz 2004;138(21):1633-43. Disponible: www.hc-sc.gc.ca/hpfb-dgpsa/tpd-dpt/sch-1272_f.html (consulté le 3 mars 2005).

3. Glasier A, Baird D. The effects of self-administering emergency contraception. N Engl 7 Med 1998;339(1):1-4.

4. Raymond EG, Chen PL, Dalebout SM. "Actual use" study of emergency contraceptive pills provided in a simulated over-the-counter manner. Obstet Gynecol 2003;102(1):17-23.

5. Soon JA, Levine M, Osmond BL, Ensom MHH, Fielding DW. Effects of making emergency contraception available without a physician's prescription: a population-based study. $7 A M C 2005 ; 172(7): 878-83$. 